\title{
Apoio matricial: dispositivo para resolução de casos clínicos de saúde mental na Atenção Primária à Saúde
}

Matrix support: device for resolution of mental health clinical cases at the Primary Health Care Apoyo matricial: dispositivo para resolución de casos clínicos de salud mental en la Atención Primaria de Salud

\author{
Maria Salete Bessa Jorge', Fernando Sérgio Pereira Sousa", Túlio Batista Franco"II \\ ' Universidade Estadual do Ceará, Centro de Ciências da Saúde, Programa de Pós-Graduação em Cuidados Clínicos em \\ Enfermagem, Programa de Pós-Graduação em Saúde Pública. Fortaleza-CE, Brasil. \\ "Universidade Federal do Piauí, Centro de Ciências da Saúde, Curso de Enfermagem. Teresina-Pl, Brasil. \\ I"' Universidade Federal Fluminense, Centro de Ciências Médicas, Instituto de Saúde da Comunidade. Niterói-RJ, Brasil.
}

\author{
Submissão: 28-08-2012 Aprovação: 13-08-2013
}

\begin{abstract}
RESUMO
O estudo objetivou compreender o apoio matricial como um dispositivo para a resolução de casos clínicos de saúde mental no âmbito da Atenção Primária à Saúde em um estudo qualitativo realizado a partir de um estudo de caso. Os dados foram coletados por meio de observação das práticas de matriciamento e analisados sob a ótica da análise de conteúdo reflexiva. Os resultados apontam para as seguintes categorias: O caminhar da usuária: dilemas e desafios; Compartilhamento de saberes: uma ampliação de olhares? A cortina se abre: passou, mas a dor ficou! e Desfecho: construção coletiva do projeto terapêutico. Evidencia-se que o matriciamento tem potência para sinalizar os caminhos para a construção de um determinado modelo de atenção à saúde que esteja articulado e sinérgico com os princípios e diretrizes do Sistema Único de Saúde.

Descritores: Saúde Mental; Rede de Cuidados Continuados de Saúde; Atenção Primária; Estudo de Caso.
\end{abstract}

\begin{abstract}
The study aimed to comprehend the matrix support as a device to resolution of clinical cases of mental health within the Primary Health Care in a qualitative study, carried out from a case study. The data were collected through observation of matrix practices and analyzed under the view of reflective content analysis. The results pointed out to the following categories: The walking of user: dilemmas and challenges; Sharing of knowledge: an expansion of views? The curtain opens itself: It passed but the pain remains! and Results: collective construction of the therapeutic project. It highlights that matrix practices has the power to sign the paths for the construction of a particular model of health care that is articulate and synergistic with the principles and guidelines of the National Health System.
\end{abstract}

Key words: Mental Health; Net of Continued Care of Health; Primary Attention; Case Study.

\section{RESUMEN}

El objetivo del estudio fue comprender el apoyo matricial como un dispositivo para la resolución de casos clínicos de salud mental en el ámbito de la Atención Primaria de Salud en un estudio cualitativo, realizado a partir de un estudio de caso. Los datos fueron recogidos a través de observación de las prácticas de matriciamento y analizados bajo la óptica del análisis de contenido reflexiva. Los resultados apuntan para las categorías: El caminar de la usuaria: dilemas y retos; Compartir saberes: ¿una ampliación de miradas? La cortina se abre: pasó, pero el dolor se ha quedado! e el Resultado: construcción colectiva del proyecto terapéutico. Se evidencia que el matriciamento tiene potencia para señalar caminos para la construcción de un modelo particular de atención de la salud que es articulada y sinérgica con los principios y directrices del Sistema Nacional de Salud. Palabras clave: Salud Mental; red de cuidados continuados de salud; atención primaria; estudio de caso. 


\section{INTRODUÇÃO}

As ações de saúde mental desenvolvidas no âmbito da Atenção Primária à Saúde (APS) exercem papel fundamental ao contribuir com seus saberes para a ampliação do potencial resolutivo das equipes, mediante a realização do apoio matricial (AM) ou matriciamento de saúde mental que tem por objetivo superar a lógica da especialização e da fragmentação do trabalho da própria área de saúde mental.

Ao se considerar o apoio matricial como dispositivo para a resolubilidade de casos clínicos de saúde mental, é preciso esclarecer acerca do entendimento que se atribui às referidas nomenclaturas. Dessa maneira, definem-se os termos dispositivos, apoio matricial e resolubilidade. "Os dispositivos seriam recursos que alteram o funcionamento das organizações [...] sendo usados para instaurar algum processo novo"(1). O apoio matricial pode ser definido como um arranjo organizacional que surge com o objetivo de ampliar a capacidade de resolubilidade das ações de saúde, ao propor uma reformulação no modo de organização dos serviços e relações horizontais entre as especialidades que passam a oferecer apoio técnico horizontal às equipes interdisciplinares de atenção primária, favorecendo assim a conexão em rede $^{(2)}$. A resolubilidade abrange aspectos relativos à demanda, à satisfação do cliente, às tecnologias dos serviços de saúde, à existência de um sistema de referência preestabelecido, à acessibilidade dos serviços, à formação dos recursos humanos, às necessidades de saúde da população, à adesão ao tratamento, aos aspectos culturais e socioeconômicos da clientela, entre outros ${ }^{(3)}$.

De acordo com o contexto, o matriciamento funciona como um dispositivo capaz de facilitar a resolubilidade da atenção psicossocial, porquanto abre caminhos para reflexões sobre a inserção de pessoas em sofrimento psíquico na comunidade.

Na condição de um dispositivo institucional recentemente incorporado pelo Ministério da Saúde como estratégia de gestão para a construção de uma rede ampliada de cuidados em saúde mental, o matriciamento rompe com a lógica de encaminhamentos indiscriminados para uma prática ancorada na corresponsabilização do cuidado. Ademais, visa produzir maior resolubilidade à atenção em saúde mental no contexto da APS ${ }^{(4)}$.

Nessa perspectiva, a justificativa da formulação de políticas para a atenção primária que envolva o cuidado em saúde mental estaria explicada e calcada no direito do usuário de encontrar em sua unidade sanitária de referência uma estratégia de acolhimento articulada com os demais dispositivos assistenciais presentes na rede de atenção ${ }^{(5)}$.

Em virtude de o apoio matricial de saúde mental se constituir um novo dispositivo em fase de implementação no Sistema Único de Saúde (SUS), torna-se de grande relevância a formulação de propostas que propiciem a análise dessa experiência. Nesse sentido, o objetivo desse estudo é compreender a constituição do apoio matricial como um dispositivo para a resolução de casos clínicos de saúde mental no âmbito da APS.

\section{CAMINHOS DA PESQUISA}

Trata-se um estudo de caso, método amplamente utilizado em pesquisas qualitativas e que deve ser aplicado quando o pesquisador tem o interesse em compreender uma situação singular, particular e que retrate a realidade de forma completa e profunda ${ }^{(6)}$.

A pesquisa foi realizada em um cenário no Nordeste do Brasil correspondente ao município de Fortaleza-CE que possui 2.505.552 habitantes, o qual possui 88 centros de saúde da família, distribuídos nas seis Secretarias Executivas Regionais (SERs), com cobertura da ESF de $42 \%$ da população.

O estudo de caso selecionado foi acolhido pela equipe da Estratégia Saúde da Família, considerada de referência para os usuários adstritos do território em discussão e composta por um médico, enfermeira e uma assistente social, bem como pela equipe do Centro de Atenção Psicossocial - CAPS Geral (equipe matriciadora), formada por um médico, enfermeiro e uma psicóloga, a partir da observação em atividade de apoio matricial de saúde mental na APS.

Para a produção de dados adotou-se a observação sistemática das práticas no matriciamento que ocorreu inicialmente com a aproximação dos campos empíricos, em que se procedeu à observação da atividade de matriciamento. Estes primeiros dados foram discutidos em devolutiva aos trabalhadores, então, sujeitos da pesquisa, para aprofundamento da compreensão em relação aos mesmos. Posteriormente novos dados foram produzidos, por continuidade da observação direta da atividade da equipe. Foi tomado um caso para estudo. Este se desenvolveu tendo os próprios trabalhadores como fonte de dados, em discussões conjuntas sobre o caso. Utilizou-se um caderno de campo para registro do cotidiano da atividade matricial.

$\mathrm{Na}$ análise do material empírico, optou-se pela análise de conteúdo, numa perspectiva crítica e reflexiva, com ênfase em eixos temáticos, por possibilitar a descrição e a explicação de um pensamento, o qual pode ser compreendido por meio da linguagem e da observação, como núcleos e instrumentos de comunicação entre os homens ${ }^{(7)}$. Essa análise se fez com a leitura do material horizontal, transversal e criação de núcleos de sentido que foram agrupados em categorias e, na sequência, procedeu-se à organização das informações contempladas nas observações, com vistas a ampliar a compreensão do fenômeno.

Cabe ressaltar que a pesquisa é um destaque do projeto intitulado "A rede de atenção primária como elo de integração da saúde mental, com ênfase no matriciamento", financiado pelo MS/FUNCAP/CNPq. O projeto foi submetido à análise do Comitê de Ética em Pesquisa (CEP) da Universidade Estadual do Ceará (UECE), com o parecer favorável do CEP/UECE, sob protocolo $\mathrm{n}^{\circ}$ 08622882-0.

\section{RESULTADOS E DISCUSSÃO}

O estudo evidencia o caso de uma usuária, $\mathrm{Ml}$, do sexo feminino, de 56 anos, separada, procedente de um bairro denominado Vila União, situado na cidade de Fortaleza-CE. Ao ser 
atendida, MI apresentou como queixa principal sintomatologia caracterizada por dores de cabeça, ansiedade, insônia, choro fácil, dependência de medicamentos para o sono regular. Segundo refere, aos 20 anos de idade manifestou os primeiros sinais de sofrimento, a qual se iniciou com a sensação de enrijecimento do corpo, sendo em seguida encaminhada para o hospital psiquiátrico. Nesta instituição foi atendida pelo médico que prescreveu medicamentos e decidiu por não interná-la. Conforme ela acrescenta, durante determinado período fez uso dos medicamentos prescritos e com o tempo interrompeu o uso por considerar que estava bem. Embora tenha sido encaminhada para o CAPS, não buscou este centro de atenção.

Posteriormente, decorrido um mês, manifestou novamente outra crise, com o mesmo quadro sintomatológico da primeira. Submeteu-se a tomografia e, no desenrolar da sua narrativa, chamou a atenção da equipe para o seguinte fato: o médico que a atendeu naquela época ironicamente a advertiu de que ela "não tinha crânio fechado", dessa maneira, continuaria sentindo mesmo fortes dores de cabeça.

Segundo MI relata, ela é mãe de dois filhos, porém reside sozinha. Nos dias atuais toma ansiolíticos. Quanto à sua ocupação, trabalha no setor administrativo de uma escola, emprego propiciado por um político da cidade onde mora. Consoante refere, sente-se pressionada constantemente pelo citado político que alega o fato de ter proporcionado a ela a sua ocupação atual. Assim, encontra-se em sofrimento mental e queixa-se de desgaste físico em consequência da situação vivenciada. Refere sentir-se pressionada constantemente pelo citado político que alega o fato de ter proporcionado a ela a sua ocupação atual. Apresenta também queixa de desgaste físico em consequência da situação vivenciada, caracterizando-se um quadro de sofrimento psíquico.

\section{O caminhar da usuária: dilemas e desafios}

Os trabalhadores da saúde do CAPS e do centro de saúde da família iniciaram a discussão do caso, procurando compreender de que maneira se deu o desenho do fluxo de atendimento da usuária no centro de saúde da família, pois, conforme entendimento, o fluxo possibilita a descrição do caminhar do usuário em busca de acompanhamento e respostas concernentes às suas necessidades de saúde. Tem também por finalidade reconhecer o processo de trabalho da equipe, revelando seus desdobramentos, as linhas de tensão e os ruídos enfrentados no caminho. Dessa maneira, identificou-se durante o atendimento que a usuária, ao acessar o serviço, foi acolhida inicialmente na recepção, onde recebeu informações acerca dos atendimentos realizados.

No concernente ao caso em análise, ao serem detectadas suas especificidades, houve o encaminhamento para a consulta de enfermagem e, nesta, ao se identificar a demanda de saúde mental, conduziu-se a usuária para avaliação com o médico clínico, pois a definição dos casos clínicos mais complexos deverá ser discutida no encontro do matriciamento no centro de saúde da família. Dessa maneira, o caso foi acompanhado de forma compartilhada no apoio matricial de saúde mental realizado pelos trabalhadores da saúde do Centro de Atenção Psicossocial e da Estratégia Saúde da Família, buscando-se assim operar o cuidado tendo como horizonte de trabalho a integralidade da atenção.

Antes da entrada da usuária, a equipe de referência, composta então por enfermeiro, médico e agente comunitário de saúde no centro de saúde da família, expõe a situação e o contexto do caso a ser discutido para a equipe de apoio matricial, formada pelo psiquiatra, assistente social e enfermeira do CAPS.

Após essa explanação, a usuária é recepcionada pela equipe interdisciplinar. Nesse momento, ela já foi esclarecida sobre o atendimento conjunto e se apresenta aos demais profissionais. O processo se desenvolve com a participação de todos os atores envolvidos, sempre sob a condução da equipe de referência, em face da maior vinculação desta com MI.

É nessa linha de entendimento que alguns estudos afirmam que a prática do matriciamento possibilita o início da mudança no fluxo burocrático e hierárquico de usuários na rede e na lógica dos encaminhamentos dos casos atendidos na ESF e nos serviços de saúde mental ${ }^{(8)}$. Isso pode ser compreendido por haver uma aproximação entre os trabalhadores desses serviços, facilitando a comunicação e a interação entre eles, tornando referência e contra referência personalizadas. Transcende-se, assim, o método tradicional do encaminhamento restrito e descompromissado.

Compartilhamento de saberes: uma ampliação de olhares?

Conforme se observa ao analisar o estudo de caso, as ações de apoio matricial em saúde mental passam por um processo de implementação. Com isso ainda é comum um relativo desconhecimento dos trabalhadores da atenção primária sobre o apoio matricial. Mesmo alguns que conhecem não se comprometem em utilizá-lo, pois o percebem como um serviço especializado, enquanto outros o entendem como sendo apenas uma proposta pedagógica, na qual não há um acompanhamento efetivo dos usuários e nem formação de uma equipe de referência eficiente.

A inexistência de práticas de saúde mental na atenção básica está associada a determinados motivos, os quais a formação dos profissionais e o pouco conhecimento acerca do processo de reforma psiquiátrica, capacitação insuficiente para atuarem junto aos casos de transtornos mentais, as péssimas condições de atendimento dos casos nas unidades básicas de saúde, a falta de medicações psiquiátricas para fornecer aos pacientes, a quase inexistência de uma rede assistencial em saúde mental de suporte, e na existência desta uma carência de entrosamento com os serviços especializados da área que funcionem com retaguarda e permitam a referência rápida quando necessário, e os conceitos ainda arraigados em um modelo asilar ${ }^{(9)}$.

Alguns desses aspectos foram encontrados durante o atendimento, como a necessidade da equipe de referência de identificar inicialmente os sinais e sintomas com vistas a propor o diagnóstico da doença. Assim, fazem inferências como: o que Ihe incomoda mais, a ansiedade, dor de cabeça? Como é essa ansiedade de que você fala? O que você sente no seu corpo que você acha que é essa ansiedade e como esse choro acontece?

Deste modo, é possível reproduzir socialmente a relação tecnicista no campo psicossocial, ou seja, redireciona-se a forma de atendimento baseada na intervenção direta na 
patologia em contraposição às demandas existenciais dos sujeitos. Visto assim, coloca-se a doença em destaque e desloca-se o olhar para ela, tornando-o objetivo de todo o trabalho; é apenas a ação técnica.

Tal fragmentação do cuidado tende a produzir um cuidado pautado nas práticas tradicionais, que refletem o "modelo produtor de procedimentos", onde a racionalidade científica e técnica é que determina a atenção á saúde. Assim, o sujeito que necessita de cuidado é reduzido à posição de objeto e fica passivo as intervenções meramente curativas ${ }^{(10)}$.

Em seguimento a essa discussão, os estudos de Oliveira ${ }^{(11)}$ realizados em Vitória-ES, refere que, dentre os avanços ocorridos com a efetivação do matriciamento, se destaca o enfrentamento que o dispositivo matricial impõe frente à superação da fragmentação do cuidado, tornando a atenção às pessoas com transtornos mentais mais resolutivas.

Ao mesmo tempo, a supremacia do modelo positivista de atenção à saúde é reforçada, também, pela usuária, pois suas colocações, quase sempre, envolvem o tratamento farmacológico e seu quadro clínico. Assim, a usuária refere: E a minha tomografia, e os meus remédios? Quero saber se eu ainda vou continuar tomando os medicamentos. Quem passou foi a doutora lá do posto e sem ele eu não consigo dormir. Olha, eu preciso de um médico me acompanhando [...].

O "saber médico" predominou nas discussões da equipe, onde "as soluções" são dadas geralmente com a indicação de algum medicamento, sem o devido acompanhamento integral do usuário.

Fundamentados nos estudos de Figueiredo e Onocko-Cam$\operatorname{pos}^{(12)}$, desenvolvidos em Campinas-SP, dentre os achados, os gestores participantes da pesquisa consideram que a atenção básica deve desenvolver o trabalho preventivo e educativo. Também deve ofertar propostas clínicas e curativas, nucleares da saúde mental, porém acredita que é preciso ainda avançar no desenvolvimento de propostas, como estimula a prática do matriciamento o aspecto integral do cuidado, buscando compreender o indivíduo como um todo, nas suas dimensões biológicas, psicológicas e sociais, em contraposição às atitudes fragmentárias e reducionistas, exclusivamente biologicistas e medicamentosas.

A excessiva demanda da usuária por medicamentos e exames, muitas vezes sem uma justificativa técnica, revela o predomínio de uma ideia muito frequente: o cuidado está associado ao alto consumo de tecnologias duras, onde sua dimensão relacional é negligenciada. Marcado por esta relação, o modo de produção do cuidado gera a denominada "produção imaginária da demanda", ou seja, uma demanda que é verdadeira, porque sentida como necessidade pela usuária; mas é também falsa, porque não é entendida tecnicamente como necessária. De modo geral, a demanda imaginária vem de uma formação sócio-histórica dos modelos de assistência desenvolvidos no século XX, fortemente centrados nos procedimentos, alimentados por práticas excessivamente prescritivas. E quanto mais os processos de trabalho estão centrados na instrumentalização do cuidado em saúde, mais a demanda imaginária se manifesta. Urge, pois, uma reestruturação dos modos de produzir o cuidado, em que os fatores acolhimento, vínculo e seguimento de projetos terapêuticos integrais sejam o centro da atividade assistencial ${ }^{(10)}$.

A nova forma de promover saúde requer ruptura com os paradigmas da clínica tradicional do modelo biomédico, efetivando-se outra forma de fazer clínica a ocorrer numa visão ampliada do processo saúde-doença-cuidado. Uma clínica na qual o objetivo da prática não é a cura, mas a produção do cuidado integral em que o sujeito é sempre biológico, social e subjetivo ${ }^{(13)}$.

Ao perceber que a condução do projeto terapêutico era, basicamente, de natureza medicamentosa, a equipe matricial procura fazer sugestões, levantamentos e intervenções dialógicas com questões sobre a subjetividade humana, na tentativa de promover uma discussão ampliada acerca dos casos, não negando o valor do medicamento, porém explorando outras ações de saúde no delineamento do projeto terapêutico. Dessa maneira, busca interrogar e colocar em reflexão se a medicação é realmente necessária, e se não há outros mecanismos e ferramentas de auxílio ao usuário, sempre no intuito de promover a sua autonomia- Então, a equipe busca uma "substitutividade" nos dispositivos terapêuticos, isto é, uma substituição dos recursos instrumentais por formas de cuidar que possam também ser eficazes, e que substituam, em algum grau, por exemplo, o uso de medicamentos.

Diante desse outro modo de cuidar do outro, a equipe de apoio matricial utiliza-se de ferramentas para a promoção da saúde mental dos usuários por meio de instrumentos que produzam acolhimento, autonomia e resolubilidade ${ }^{(14)}$.

Como observado, o compartilhamento de saberes com os profissionais do CAPS possibilita se alargar a visão da equipe da ESF, particularmente ao sugerir aspectos além dos cuidados com os sinais e os sintomas. Desse modo, inclui os aspectos da vida e do sofrimento apresentados no caso clínico mediante vivências pessoais e da própria existência humana, na tentativa de ampliação do projeto terapêutico dos usuários.

Assim, o apoiador matricial é um especialista detentor de um núcleo de conhecimento e de um perfil distinto daquele dos profissionais de referência; contudo, ele pode agregar recursos de saber e mesmo colaborar com intervenções que aumentem a capacidade em dar resolubilidade a problemas de saúde da equipe primariamente responsável pelo caso. $\mathrm{O}$ apoio matricial procura construir e avivar espaço para a comunicação ativa e para o compartilhamento e aprimoramento de conhecimento entre profissionais de referência e apoiadores. Mediante encontros recorrentes discutem-se casos ou problemas de saúde selecionados pela equipe de referência e procura-se elaborar projetos terapêuticos integrados e acordar linhas de intervenção para os vários profissionais envolvidos ${ }^{(15)}$.

Entre as equipes de referência e matricial se produz um espaço de interseção no qual ocorre o inusitado encontro de saberes, práticas, e afetos, que neste processo produzem algo em comum: o cuidado. Consoante se evidencia, entre as equipes é preciso haver mais do que um encontro no qual se realiza troca de tecnologias de intervenção; entre elas deve se construir, também, identidades, no sentido atribuído ao cuidado, na forma com que significam o usuário e seu sofrimento. Esta dimensão, sensível, de percepção da produção do cuidado, é capaz de tornar o processo de trabalho mais eficaz 
quer para o usuário, quer para o próprio trabalhador da saúde, que se sente potente à medida que os efeitos da sua prática são verificados como resolutivos.

Como se percebe, as interações entre as duas equipes equipe de apoio matricial e equipe de referência - favorecem a mudança de posicionamentos na forma de abordar e acolher o sofrimento do outro, ancorando-se no olhar psicossocial estimulado pelos trabalhadores da saúde mental, os quais propõem ampliar a discussão/reflexão, com ênfase nos aspectos da subjetividade. Dessa maneira, rompem com a lógica restrita do binômio queixa/conduta. De modo enfático, revela-se: o medicamento corresponde a mais um item do projeto terapêutico, mas não é o mais importante.

Em corroboração a essa ideia, o apoio matricial modifica o foco da orientação da assistência, que se desloca progressivamente dos serviços e seus cardápios de programas e ofertas, para se dar a partir das necessidades dos sujeitos, das famílias, do território e da rede de relações que nele acontecem $^{(12)}$.

\section{A cortina se abre: passou, mas a dor ficou!}

Em continuidade à descrição do seu caso, a usuária, agora mais à vontade com a equipe, a denotar o início do vínculo, relata um episódio possivelmente potencializador de suas crises.

Vou chegar numa coisa agora [...] a minha filha quando eu tive, naquele tempo era no interior, um lugar pequeno, e aí a pessoa perder a virgindade e engravidar na adolescência naquela época era um absurdo. Hoje as coisas vão mudando e tá mais fácil [...]. Então assim meu pai, que era um homem ignorante, violento e ele não aceitou e me obrigou a doar a minha filha e isso foi uma coisa que me chocou muito [...], um trauma que eu passei dezesseis anos para poder pensar em ter o segundo filho. E essa filha eu não criei [...], porque ele me obrigou a dar e isso é um trauma muito grande. Eu não sei se perdoei [...] mas assim era meu pai. Passou, mas a dor ficou! Ficou a cicatriz! Não sei nem como eu consegui viver depois disso.

De acordo com o observado, o fato produziu o efeito, na usuária, fixando-a em um território existencial no qual ela aparece como "mãe-abandônica", "filha-transgressora". Isto gerou nela mesma uma subjetividade na qual há intensa despontencialização da vida, uma reduzida potência vital, ocorrendo, pois, uma produção de si, centrada na sua própria mortificação, representada pela dor e sofrimento subsequentes. É assim que parece acontecer, tomando por referência a ideia segundo a qual os encontros que causam tristeza reduzem a capacidade de agir no mundo ${ }^{(16)}$, e, no caso, o encontro da mãe com a ordem paterna, com o abandono, com a perda, reduziu por completo sua capacidade de agir. Portanto, resultou em produção de morte em si mesmo, manifestada pelo intenso sofrimento.

Conforme mencionado, o vínculo, tal como outros fatores, deve ser o centro da atividade assistencial. $O$ vínculo é uma ferramenta que agencia as trocas de saberes entre o técnico e o popular, o científico e o empírico, o objetivo e o subjetivo, a razão e os afetos, convergindo-os para a realização de atos terapêuticos conformados a partir das sutilezas de cada coletivo e de cada indivíduo. Ele favorece outros sentidos para a integralidade da atenção à saúde $\mathrm{e}^{(17)}$. O trabalho em saúde deve ser permeado pelos encontros diversos e pelas múltiplas visões na relação entre o trabalhador e o usuário. Por outro lado, a relação terapêutica também é constituída pela dor, sofrimento, vivências e percepções de vida em que os saberes e práticas no campo da saúde mental precisam estabelecer mecanismos para tornar evidente os elementos assistenciais, subjetivos e sociais ${ }^{(18)}$.

A privação ou a ausência da relação afetiva/vínculo mãe-filho observada no caso clínico citado, é decisiva para a saúde mental desses indivíduos ${ }^{(19)}$. Ademais, várias formas de neuroses e desordens de caráter, sobretudo psicopatias, podem ser atribuídas seja à privação do cuidado materno, seja à descontinuidade na relação da criança com uma figura materna durante os primeiros anos de vida.

\section{Desfecho: construção coletiva do projeto terapêutico}

Ao serem esclarecidas as dúvidas e explorado todo o contexto, solicitou-se à usuária esperar em outra sala, enquanto as equipes promoviam uma nova discussão/reflexão do caso.

Assim, a usuária aguarda a discussão da equipe e ao fim de alguns minutos retorna à sala e tem a oportunidade de decidir e pactuar com a equipe o seu projeto terapêutico, o qual vai se desenhando em forma de esboço, receptivo a possibilidades e interações de diferentes saberes.

Durante a exposição de um caso clínico no matriciamento, nota-se que os participantes colocam-se no lugar do outro, oferecem-se como parceiros, dão sugestões de conduta baseadas em sua experiência profissional e pessoal(20). Assim, constrói-se um saber novo e híbrido, composto de diversas visões e experiências. Sabe-se que cada situação tem suas particularidades e não há uma receita a ser seguida na construção do projeto terapêutico. Entretanto, ao longo do trabalho, observa-se claramente a amplitude de conhecimento que cada participante da equipe adquire.

Compreende, portanto, que o projeto terapêutico trata de uma discussão prospectiva de caso, em que, depois de uma avaliação de risco e de vulnerabilidade compartilhada, são acordados procedimentos de cuidado a cargo de diversos membros da equipe, no qual o grupo incorpora a noção interdisciplinar e recolhe a contribuição das várias especialidades e de distintas profissões ${ }^{(15)}$.

Desse modo, o projeto terapêutico é elaborado com base nas necessidades de saúde de cada usuário, não excluindo suas opiniões, seus sonhos, seu projeto de vida. Esse projeto é algo singular, uma interação democrática e horizontal entre trabalhador/usuário/família. Por isso, pode seguir vários caminhos.

No diálogo sobre a construção do projeto terapêutico, a equipe compartilhou com a usuária todas as possibilidades e etapas desse projeto, com vistas a elaborá-lo de maneira 
conjunta, pois desde o início a equipe tentou estimular o protagonismo da usuária. Nesse contexto, sugeriu diferentes possibilidades de atos em saúde que poderiam compor a planificação do projeto terapêutico, tais como a psicoterapia individual, em que a argumentação e a explicação de tal atividade se baseou na ideia de facultar a ressignificação dos sentimentos ainda emergentes geradores de sofrimentos.

O matriciamento se configura como um elo entre a atenção primária e os CAPS, tendo em sua essência o compartiIhamento de experiências e de saberes, a corresponsabilização dos casos, buscando oferecer múltiplos caminhos para a efetivação do projeto terapêutico ${ }^{(8)}$. Assim, o usuário poderá optar pelo percurso que considerar mais viável.

Ressalta-se, porém que é preciso que toda a equipe considere o projeto terapêutico do usuário como algo flexível. Nessa ótica, ele deve ser reavaliado periodicamente, porquanto as necessidades do sujeito se modificam, sendo sempre dinâmico.

Nesse prisma, a partir das pactuações e articulações entre profissionais surgiu à proposta da psicoterapia individual como forma de ensejar a discussão/reflexão dos sentimentos da usuária no tocante ao seu sofrimento, bem como os valores e desejos por ela manifestados em relação ao plano de cuidados inicialmente proposto. Contudo, a usuária se mostrou resistente à indicação da psicoterapia. Outro ponto relevante coloca em evidência a questão da corresponsabilização da usuária no tratamento, pois esta se mostrou receptiva, principalmente, à ideia farmacológica. Com isso, a irredutibilidade da usuária em relação às outras formas de cuidado ocasionou desconforto por parte da equipe em propor outras opções de tratamento.

A equipe começa a se perguntar "se a usuária possuía potência naquele momento para decidir sobre qual a meIhor estratégia de cuidado a seguir"; ou "se ela apresentava potencial de gestão para conduzir e tomar decisão sozinha". Contudo, fica claro que a equipe precisaria propiciar outros momentos de escuta qualificada para a referida usuária, e nestes momentos verificar o melhor projeto terapêutico. Poderia, então, no campo das tecnologias leves, abrir o leque de alternativas para além da própria psicoterapia, e negociar com a usuária dispositivos terapêuticos adicionais à medicação, até a sua redução e extinção. Mas para isto a própria equipe deve ampliar suas opções de cuidado no intuito de proporcionar à usuária um rol de possibilidades, operando, portanto mais sintonizada com a clínica dos afetos, a qual procura enfatizar o lado sensível, a possibilidade de proporcionar bons encontros, afecções capazes de recuperar a alegria, retomando Espinosa, para que a usuária produza em si mesma uma nova e revigorada potência de agir no mundo ${ }^{(16)}$.

Como observado, as equipes dos CAPS têm se mostrado bastante ativas e preocupadas em estabelecer o matriciamento de forma real nas unidades de saúde, e, apesar dos constantes desafios, vêm buscando alargar as oportunidades de se realizar a clínica ampliada e integração dialógica entre distintas especialidades e profissões. Cabe-lhes o desafio de transformar a reorganização do processo de trabalho em saúde das equipes a partir do dispositivo do Apoio Matricial e da reflexão sobre a prática em saúde.

Nesta perspectiva, a mobilização de componentes afetivos contribui para a determinação das atitudes profissionais, onde desenvolvem a empatia e a compreensão do paciente como um ser humano.

\section{CONSIDERAÇÕES FINAIS}

O apoio matricial faculta o encontro com o outro e com o campo saúde mental rico em possibilidades, porquanto questões antes não percebidas pelos profissionais, motivadas por receio e estigma de enxergar a demanda de saúde mental na atenção básica, tornam-se mais uma necessidade de saúde que é encarada com um novo olhar pela equipe. Desse modo, evitam-se os encaminhamentos desnecessários e o usuário passa a ter um vínculo maior com a equipe, o qual favorece sua autonomia e seu modo de andar na vida.

No caso analisado, o vínculo propicia o discurso de uma experiência de abandono, fixação em um território existencial em que suas identidades são marcadas por personagens vinculadas a esta questão. Esta situação gera na usuária uma intensa e crônica vivência de sofrimento, "Passou, mas a dor ficou!". Inegavelmente, o cuidado em saúde desenvolvido pela articulação entre as equipes aponta para uma tentativa de reelaborar esse modo de andar na vida. Evidencia-se, contudo, a necessidade de ampliar a oferta de terapêuticas para melhor vinculação da usuária e/ou alargar o tempo de escuta e diálogo com ela com vistas à maior resolubilidade. Isto, porém, pode ser insuficiente, pois as atividades terapêuticas precisam operar uma desterritorialização do lugar onde a usuária se encontra, da "mãe-abandônica" e "filha-transgressora", para outra expressão de si mesma. Para tal, urge formar um novo território existencial, o qual deve se constituir em substituição ao atual, o que ressignificaria a usuária para ela mesma. Conforme se percebe, entretanto, a equipe não detém tecnologias de cuidado para operar um projeto terapêutico com esta radicalidade, mas busca um caminho nesta direção, o de ampliar a escuta, pois desta atitude podem surgir novas e inovadoras possibilidades.

O dispositivo equipe matricial pode contribuir fundamentalmente para a constituição da outras ferramentas da equipe, para o cuidado em saúde mental. Ele tem potência para problematizar as práticas, agregar novos dispositivos, sinalizar os caminhos para a construção de outro modelo de assistência à saúde que esteja articulado e sinérgico com os princípios e diretrizes do SUS e, sobretudo processos de cuidado baseados em múltiplos dispositivos. Para tanto, faz-se imprescindível a organização dos processos de trabalho em função da produção do cuidado como finalidade do trabaIho em saúde, que investe nas tecnologias leves, na clínica dos afetos, e na corresponsabilização como forma de estimular o compromisso de trabalhadores, usuários e familiares com a produção da saúde. 


\section{REFERÊNCIAS}

1. Furtado JP. Um método construtivista para a avaliação em saúde. Ciênc Saúde Coletiva 2001;6(1):165-181.

2. Campos GWS. Equipes de referência e apoio especializado matricial: um ensaio sobre a reorganização do trabaIho em saúde. Ciênc Saúde Coletiva 1999;4(2):393-403.

3. Turrini RNT, Lebrão ML, Cesar CLG. Resolutividade dos serviços de saúde por inquérito domiciliar: percepção do usuário. Cad Saúde Pública 2008;24(3).

4. Ministério da Saúde. Departamento de Ações Programáticas Estratégicas. Saúde mental no SUS: os centros de atenção psicossocial. Brasília, DF: Ministério da Saúde; 2004.

5. Silveira DP, Vieira ALS. Saúde mental e atenção básica em saúde: análise de uma experiência no nível local. Ciênc Saúde Coletiva 2009;14(1):139-148.

6. Lüdke $M$, André ME DA. Pesquisa em educação: abordagens qualitativas. São Paulo: EPU; 1986.

7. Minayo MCS. O desafio do conhecimento - pesquisa qualitativa em saúde. 11. ed. São Paulo: HUCITEC; 2008.

8. Sousa FSP, Jorge MSB, Vasconcelos MGF, Mont'alverne Barros MM. Tecendo a rede assistencial em saúde mental com a ferramenta matricial. Physis: Rev Saúde Coletiva 2011;21(4):1579-1599.

9. Nunes M, Juca VJ, Valentim CPB. Ações de saúde mental no programa saúde da família: confluências e dissonâncias das práticas com os princípios das reformas psiquiátrica e sanitária. Cad Saúde Pública 2007;23(10):2375-2384.

10. Franco TB, Merhy EE. A Produção imaginária da demanda e o processo de trabalho em saúde. In: Pinheiro R, Mattos RA, organizadores. Construção Social da Demanda: direito à saúde, trabalho em equipe, participação e espaços públicos. Rio de Janeiro: ABRASCO; 2005. p.1-16.
11. Oliveira GN. Devir apoiador: uma cartografia da função apoio. Campinas. Tese [Doutorado em Saúde Coletiva]Universidade Estadual de Campinas; 2011.

12. Figueiredo MD, Onocko-Campos R. Saúde Mental na atenção básica à saúde de Campinas, SP: uma rede ou um emaranhado?. Ciênc Saúde Coletiva 2009;14(1):129-138.

13. Campos GWS. Saúde paidéia. São Paulo: Hucitec; 2003.

14. Jorge MSB, Pinto DM, Quinderé PHD, Pinto AGA, Sousa FSP, Cavalcante CM. Promoção da Saúde Mental - Tecnologias do Cuidado: vínculo, acolhimento, corresponsabilização e autonomia. Ciênc Saúde Coletiva 2011;16(7):3051-3060.

15. Campos GWS, Domitti AC. Apoio matricial e equipe de referência: uma metodologia para gestão do trabalho interdisciplinar em saúde. Cad Saúde Pública 2007;23(2):399-407.

16. Deleuze G. Espinosa: filosofia prática. São Paulo: Escuta; 2002.

17. Santos AM, Assis MMA, Nascimento MAA, Jorge MSB. Vínculo e autonomia na prática de saúde bucal no programa saúde da família. Rev Saúde Pública 2008;42(3):464-70.

18. Fonseca $A F$, Corbo $A D^{\prime} A$, organizadores. O território e o processo saúde-doença. Rio de Janeiro; EPSJV/Fiocruz; 2007.

19. Bowlby J. Cuidados maternos e saúde mental. 5. ed. São Paulo: Martins Fontes; 1995.

20. Delfini PSS, Sato MT, Antoneli PP, Guimarães PS. Parceria entre CAPS e PSF: o desafio da construção de um novo saber. Ciênc Saúde Coletiva 2009;14(suppl 1):1483-1492. 